Artículo de investigación.

Cómo citar: L. Moya, D. Álvarez, y S. Meneses. "Estudio exploratorio de medición de material particulado respirable en biciusuarios de Engativá, Bogotá”. Inventum, vol. 15, no. 29, pp. 37-48. doi: 10.26620/uniminuto.inventum.15.29.2020.37-48

Editorial: Corporación Universitaria Minuto de Dios - UNIMINUTO.

ISSN: $1909-2520$

eISSN: 2590-8219

Fecha de recibido: 31 de mayo de 2020 Fecha de aprobado: 15 de junio de 2020 Fecha de publicación: 15 de julio de 2020

Conflicto de intereses: los autores han declarado que no existen intereses en competencia.

\section{Estudio exploratorio de medición de material particulado respirable en biciusuarios de Engativá, Bogotá}

\section{Exploratory study to measure respirable particulate material in bi-users in Engativá, Bogotá}

\author{
Estudo exploratório para medir \\ material particulado respirável em \\ biusuários em Engativá, Bogotá
}

\begin{abstract}
Resumen
En Bogotá se realizan aproximadamente 900.000 viajes en bicicleta a diario, siendo este un medio de movilidad sostenible y amigable con el ambiente. Los usuarios que utilizan este medio de transporte se exponen a una deficiente calidad del aire, que ha traído como consecuencia altos niveles de enfermedades respiratorias agudas, generadas por la exposición a elevadas dosis de material particulado que se encuentra suspendido en el aire.
\end{abstract}

En la Universidad Libre sede Bosque Popular, aproximadamente 500 estudiantes, docentes y administrativos se movilizan en bicicleta, de los cuales un 60 \% transitan por la ruta que va desde el barrio Bonanza, Avenida Medellín (calle 80), avenida Rojas, hasta la Universidad; lugar que no cuenta con una ciclorruta y por lo tanto los biciusuarios se encuentran expuestos a respirar más cantidades de este contaminante.

El presente trabajo se centra en el análisis, modelado de los resultados obtenidos de la medición de material particulado respirable por los biciusuarios en su trayecto cotidiano en la ruta anteriormente mencionada y la comparación con los límites permisibles, lo que permite identificar los niveles de riesgo a los cuales ellos están expuestos. La complejidad de este experimento radica en el "ambiente" de toma de muestras, entendido este como las rutas de movilidad ya que son espacios abiertos donde existe una serie de aspectos no controlados en contraste con los muestreos de higiene en espacios laborales normales y la aplicación de un estudio de higiene industrial llevado al tema de movilidad en bicicleta, el cual no se ha realizado antes.

Palabras clave: ciclistas, higiene industrial, material particulado, movilidad, riesgo.

\begin{abstract}
In Bogotá approximately 900,000 bicycle trips are made daily, this being a sustainable and environmentally friendly means of mobility. Users who use this means of transport are being exposed to poor air quality, which has resulted in high levels of acute respiratory diseases, generated by
\end{abstract}


exposure to high doses of particulate material that is suspended in the air, as a result of emissions from fixed and mobile sources.

At the Universidad Libre, Bosque Popular headquarters, approximately 500 students, teachers and administrators move by bicycle, of which $60 \%$ travel on the route from Bonanza Medellin Avenue(80 street), Rojas Avenue, to the University, that way does not have a bike path and therefore cyclists are exposed to breathing more amounts of this pollutant.

The present work focuses on the analysis, modeling of the results obtained from the measurement of particulate material breathable by cyclists in their daily journey route previously mentioned and the comparation with the permissible limits, in order to identify the risk levels to which cyclists are exposed. The complexity of this experiment lies in the sampling "environment", understood as mobility routes since they are open spaces where there are a number of uncontrolled aspects in contrast to hygiene sampling in normal work spaces and the application of an Industrial hygiene study carried out on the topic of bicycle mobility, which has not been carried out before

Keywords: cyclists, industrial hygiene, particulate material, mobility, risk.

\section{Resumo}

Em Bogotá, cerca de 900.000 viagens de bicicleta são feitas diariamente, sendo este um meio de mobilidade sustentável e ecológico. Os usuários que utilizam esse meio de transporte estão sendo expostos à má qualidade do ar, o que resulta em elevados índices de doenças respiratórias agudas, geradas pela exposição a altas doses de material particulado em suspensão no ar.

$\mathrm{Na}$ universidade gratuita Bosque Popular, cerca de 500 alunos, professores e administradores viajam de bicicleta, dos quais $60 \%$ percorrem o trajeto que vai do bairro Bonanza, Avenida Medellín (Calle 80), Avenida Rojas, até a Universidade; Local que não possui ciclovia e por isso os biusuários ficam expostos a respirar mais quantidades desse poluente.

Este trabalho tem como foco a análise, modelagem dos resultados obtidos na medição de material particulado respirável por biusuários em sua jornada diária no referido trajeto e a comparação com os limites permitidos, identificando os níveis de risco aos quais eles são expostos. A complexidade desta experiência reside no "ambiente" de amostragem, entendido como as vias de mobilidade por serem espaços abertos onde existe uma série de aspectos não controlados em contraste com as amostras de higiene nos espaços normais de trabalho e na aplicação de um estudo de higiene industrial sobre a questão da mobilidade em bicicletas que não foi feito antes.

Palavras-chave: ciclistas, higiene industrial, material particulado, mobilidade, risco. 


\section{INTRODUCCIÓN}

En los últimos años, Colombia ha hecho énfasis en el uso de la bicicleta [1]. La localidad de Engativá es una zona con bastante flujo de biciusuarios, al año se realizan aproximadamente 64.500 viajes en bicicleta [2]. Sin embargo, se desconocen los riesgos a los cuales están expuestos los biciusuarios en su proceso de movilidad, dado que deben realizar actividad física con mucha proximidad a las fuentes de emisión vehicular, que pueden ser de tipo público o privado [3], [4].

La Organización Mundial de la Salud (OMS) señala que, en el año 2016, el 91 \% de la población vivía en lugares donde no se respetaban las directrices sobre la calidad del aire, lo que provocaba de forma indirecta 4.2 millones de muertes prematuras al año por enfermedades cardiovasculares y respiratorias, debido a la contaminación atmosférica y la exposición a material particulado (PM2.5) [5].

Los crecientes flujos de tráfico vehicular generan altos niveles de contaminantes, lo que expone a los ciclistas a concentraciones más altas de PM10 [6]. Por esta razón es fundamental estudiar los corredores viales por donde transitan los biciusuarios, así como también las diferentes características propias de la ruta que pueden afectar el muestreo. Diferentes estudios permiten evidenciar la influencia que tienen las variablesclimáticas paraalterarel destino, transporte y concentraciones de los diferentes contaminantes como el PM10 [7] y el ozono [8].

[9] estudió las concentraciones de material particulado respirable en tres diferentes medios de transporte: bicicleta, carro y bus, estos se realizaron cuando más se presentaba flujo vehicular en horas de la mañana y tarde, como resultado obtuvo que los ciclistas sobre la carretera estaban expuestos a niveles altamente significativos de PM10, CO y UFP.

En estudios realizados en [10] se hicieron mediciones todos los días de la semana en un horario de 7:00 a. m. a 10 a. m., y se monitorearon las concentraciones de PM2.5 y Black Carbon (BC) en 4 ciclorrutas de Bogotá. Este ha sido el único estudio que incluye los fines de semana dentro de sus mediciones y que como resultado obtuvo una diferencia significativa de las concentraciones de estos contaminantes con respecto a los días entre semana.

En el año 2011, [11] realizó mediciones de MP10 en la ciudad de Bogotá, en dos lugares con presencia de ciclorruta en vías con alto tráfico vehicular. Se hizo el cálculo de dosis respirables diarias de PM10 con diferentes modos de esfuerzos y se obtuvo como resultado que un ciclista con esfuerzo alto podría inhalar dosis de PM10 entre $45 \%$ y $64 \%$ mayor que al realizarse con un esfuerzo bajo. Este estudio permitió determinar la importancia del esfuerzo y la intensidad del ejercicio en el proceso de desplazamiento de los ciclistas.

El nivel del material particulado respirable de los biciusuarios es un tema que debe medirse, ya que cada año aumenta el número de personas que utilizan la bicicleta como medio de transporte, lo que convierte al biciusuario en un actor vial significativo en la movilidad de la ciudad. Esta medición contempla aspectos complejos de la toma de muestras, así como aspectos técnicos relacionados con las metodologías y técnicas para definir este nivel.

El objetivo de este proyecto fue realizar la medición de material particulado respirable mediante métodos de higiene industrial aplicada, tales como ACGIH y toma de muestras con la norma NIOSH 600, con el uso de protocolos de laboratorio y muestreo, para identificar si las variables climáticas y las características propias de la ruta tenían incidencia en la exposición de los biciusuarios a altos niveles de concentración de material particulado.

\section{METODOLOGÍA}

Para lograr el objetivo de la investigación se realizó un análisis exploratorio mediante el cálculo de medidas estadísticas descriptivas y la elaboración de diferentes modelos para visualizar el comportamiento de los datos.
1. Toma de muestras y registro en bitácoras de variables climáticas de la ciudad.
2. Análisis estadistico de los datos (modelo exponencial y lineal multivariado)
3. Análisis de datos por el modelo de dinámica de sistemas.
4. Comparación de los modelos obtenidos

Figura 1. Metodología de la investigación. Fuente: elaboración propia. 
La investigación realizada es de tipo exploratoria, debido a que se determinará la concentración de MP10 que diariamente respiran los biciusuarios en el corredor vial comprendido entre el barrio Bonanza y la Universidad Libre en Bogotá, mediante el método de análisis gravimétrico, el cual permite conocer la masa total de la muestra por diferencia de peso del filtro de membrana, antes y después de su recolección.

Se tomó como referencia la norma UNE-EN 689, válida para exposiciones uniformes, la cual se fundamenta en obtener un número de mediciones que abarquen un mínimo del $25 \%$ del tiempo de exposición, según la siguiente tabla.

\begin{tabular}{|l|l|c|c|}
\hline Duración muestra & Ejemplos según el tipo de medición & $\begin{array}{c}\text { No. muestras } \\
\text { para 8 horas (al 25\%) }\end{array}$ & $\begin{array}{c}\text { No. mínimo de muestras } \\
\text { recomendados }\end{array}$ \\
\hline 1 minuto & Tubos detectores de corta duración & 20 \\
\hline 5 minutos & (Tubos colorimétricos) & 120 & 12 \\
\hline 15 minutos & $\begin{array}{l}\text { Tubos detectores de corta duración } \\
\text { Tubos adsorbentes } \\
\text { (carbón activado, gel) }\end{array}$ & 24 & 4 \\
\hline 30 minutos & $\begin{array}{l}\text { Tubos adsorbentes } \\
\text { (carbón activado, gel). }\end{array}$ & 8 & 3 \\
\hline 1 hora & Filtros de Muestreo & 4 & 2 \\
\hline
\end{tabular}

Tabla I. Mínimo número de mediciones por jornada según el tipo de medición Fuente: Norma UNE-EN 689.

Se tomaron muestras por ciclos, en este caso tres por ruta con duración de recorrido de 45 minutos cada una, donde cada biciusuario representa un ciclo completo de trabajo.

\section{A. Tamaño y duración de la muestra}

Se aplicó un método probabilístico sencillo para el cálculo del número de muestras a realizar, el cual se presenta a continuación:

$$
n=\frac{z^{2} P Q N}{e^{2}(N-1)+z^{2} P Q}
$$

Donde:

$Z$ = nivel de confianza

$\mathrm{P}=$ probabilidad de éxito

$\mathrm{Q}=$ probabilidad de fracaso

$\mathrm{N}=$ población de ciclistas $=500$

$E=$ nivel de error muestral

$n=218$ muestras
Sin embargo, al momento de definir la duración de cada una de las muestras se tuvo en cuenta un tiempo máximo para evitar sobrecargar los filtros y en función de la concentración, se aplicó la siguiente fórmula:

$$
T=\frac{A}{a} \times \frac{L}{C e} \times \frac{I}{r}
$$

Donde:

T: duración de las muestras

L: carga del filtro requerido en fibras/campo

A: área efectiva del filtro en $\mathrm{mm}^{2}$

a: área de retícula en $\mathrm{mm}^{2}$

$\mathrm{r}$ : caudal en $\mathrm{cm}^{3} / \mathrm{min}$

Ce: concentración esperada

De acuerdo con esto y según la norma NIOSH 600, la cual nos indica que para un límite de 8 horas y con un caudal de $2 \mathrm{~L} /$ minuto establecido se obtiene que la duración de la muestra debe ser de $t=129$ minutos. Este tiempo estará repartido en 3 muestras definidas por ruta como admisibles 
para el proyecto, lo que indica que cada muestreo tiene una duración mínima de 43 minutos para la recolección de material particulado.

\section{B. Medición}

Los muestreos se realizaron de lunes a viernes en un horario de 6:00 a. m. a 7:00 a. m. cuando el flujo vehicular era alto en la zona. Se utilizó un equipo GilAir, con ciclón, filtro y porta filtros, todo previamente calibrado de acuerdo con los parámetros establecidos en la norma NIOSH 600 [12].

\section{Preparación, toma y analisis de las muestras}

Con el fin de disminuir los niveles de errores que se pudieran presentar en los distintos momentos de manipulación de las muestras, se estableció una serie de protocolos en donde se abarcaba desde la preparación de los equipos en el labora torio, la toma de muestra en campo y el pesaje final de la muestra. Los protocolos que se elaboraron y siguieron se enumeran a continuación:
1. Protocolo de alistamientos de muestras.

2. Protocolo de alistamiento de máquinas y procesos para el experimento.

3. Protocolo de toma de muestras.

4. Protocolo de custodia y no contaminación de muestras.

5. Protocolo de registro e identificación de aspectos disturbadores.

6. Protocolo de registro de datos iniciales (pesos, bitácoras, variables meteorológicas).

\section{Ubicación de la zona de estudio}

Se realizaron varios recorridos previos con el fin de identificar aquellas características propias de la ruta, como: tiempo de recorrido, fuentes fijas y móviles de emisión presentes en la zona, estado de la vía, entre otras.

La ruta seleccionada fue: barrio Bonanza Avenida Medellín (calle 80) - Avenida Rojas - Universidad Libre sede Bosque Popular, la cual cuenta con una extensión de $5 \mathrm{~km}$ y no tiene ciclorruta.

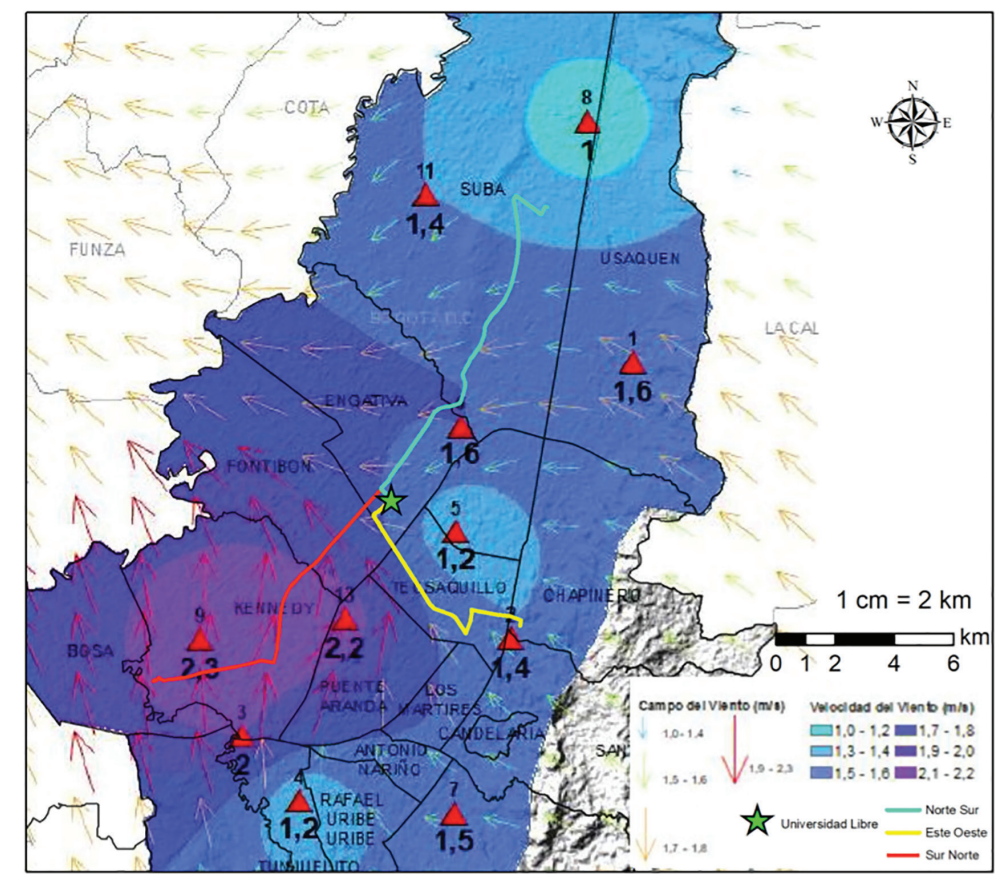

Figura 2. Mapa de ruta y dirección del viento del muestreo.

Fuente: G. Sánchez, Datos del proyecto. Universidad Libre, Bogotá, 2019. [13]. 


\section{E. Variables}

Se determinó la concentración y el nivel de exposición a material particulado PM10 en el corredor vial seleccionado donde se movilizan el $60 \%$ de los biciusuarios de la universidad. Se registraronvariables externascomola temperatura, humedad, velocidad del viento, temporada seca o de lluvia, entre otras.

Debido a que el tiempo de exposición fue inferior a 8 horas, se ajustó al valor de la norma por medio de un factor de correlación y así determinar un valor de exposición promedio.

$$
\text { Factor corrección }=\frac{8 \text { horas }}{\text { Número horas reales }} *\left(\frac{[24 \text { horas }- \text { Número horas reales }]}{16}\right)
$$

Para hacer una comparación de las concentraciones de material particulado con la normatividad de exposición ocupacional se utilizó la siguiente expresión:

\section{Valor real norma $=$ Factor corrección ${ }^{*}$ Límite de exposición Norma (OSHA ó ACGIH).}

Se realizó un ajuste para las horas reales de exposición a concentración de material particulado como se muestra en la siguiente ecuación:

$$
\begin{aligned}
& \begin{array}{l}
\text { Concentración promedio PM10 } \\
\text { Concentración PM10 * Número horas reales }
\end{array} \\
& =\frac{8}{}
\end{aligned}
$$

\section{RESULTADOS}

Las variables que se incluyen en este análisis son: concentración de material particulado con $\mathrm{ug} / \mathrm{m}^{3}$, promedio porcentual de la humedad, la temperatura en
${ }^{\circ} \mathrm{C}$, velocidad del viento en $\mathrm{km} / \mathrm{h}$, se tiene en cuenta que las mediciones fueron realizadas 35 veces, tanto en temporada de lluvia como en temporada seca.

\begin{tabular}{|l|c|c|c|c|c|c|}
\hline \multicolumn{1}{|c|}{ Variable } & Mín & Q1 & Mediana & Media & Q3 & Máximo \\
\hline Humedad & 74 & 76 & 83 & 82 & 85 & 97 \\
\hline Temperatura & 9 & 11 & 12 & 11.73 & 13 & 15 \\
\hline Velocidad del viento & 5 & 5.2 & 6 & 6.15 & 7 & 7.8 \\
\hline Concentración de MP & 0 & 1.89 & 3.738 & 5.422 & 7.345 & 30.145 \\
\hline
\end{tabular}

Tabla II. Estadísticos descriptivos de las variables

Fuente: elaboración propia, 2020. 
A continuación, se presentan los modelos que pretenden explicar los valores de material particulado respirable de los biciusuarios.

\section{A. Análisis del nivel de riesgo}

Para identificar el nivel de riesgo al cual se encuentra expuesto el biciusuario se realizó una carta de control [14], donde se pudo evidenciar el comportamiento de los datos durante los 70 días de medición, para buscar así un punto de comparación con respecto a la normativa, que de según el Icontec el límite establecido de exposición para una duración de 8 horas sin que existan afectaciones a la salud debe ser de un máximo de $5 \mu \mathrm{g} / \mathrm{m}^{3}$ [15], [16].

\section{Riesgo tolerable de material particulado}

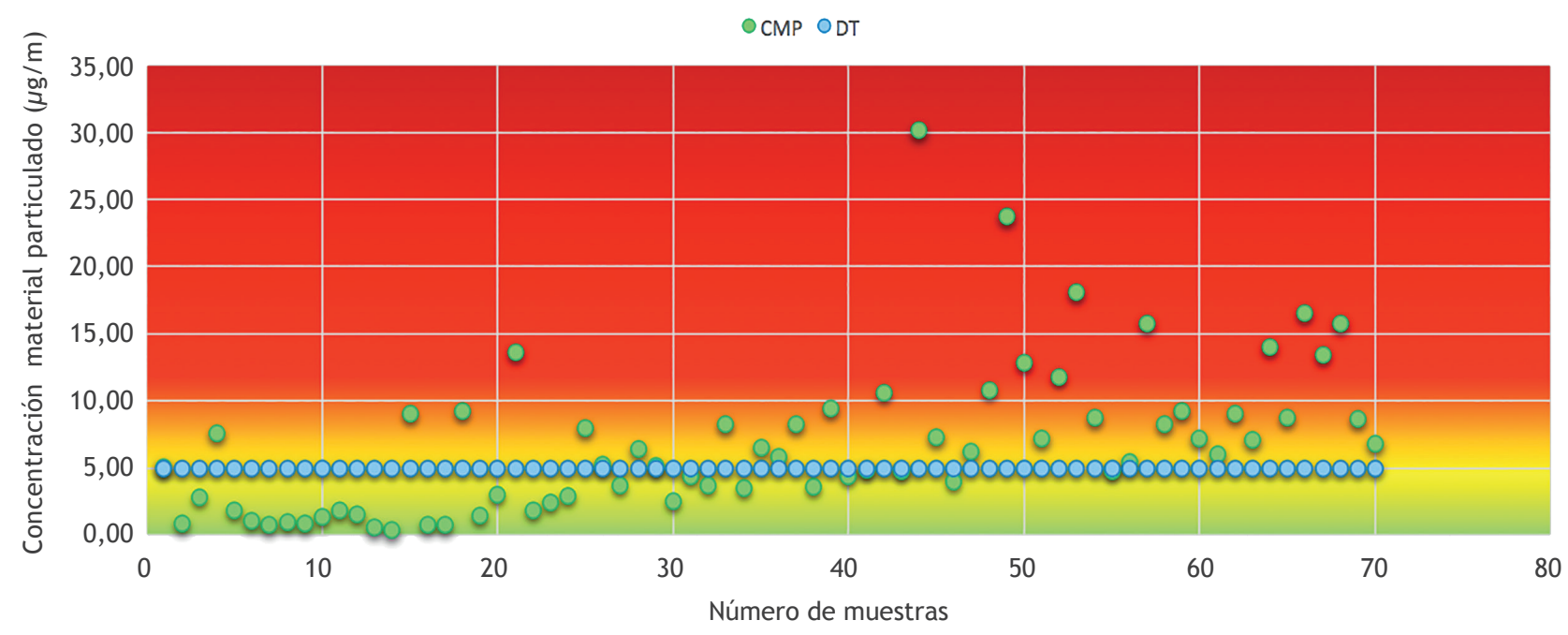

\begin{tabular}{|c|c|}
\hline NIVEL DE RIESGO & $\%$ \\
\hline Sin Riesgo & $43 \%$ \\
\hline En riesgo $(5 \mu \mathrm{g} / \mathrm{m} 3<\mathrm{x}<6 \mu \mathrm{g} / \mathrm{m} 3)$ & $9 \%$ \\
\hline En alto riesgo $>6 \mu \mathrm{g} / \mathrm{m} 3$ & $49 \%$ \\
\hline
\end{tabular}

Figura 3. Carta de control de riesgo.

Fuente: elaboración propia, 2020.

\begin{tabular}{|l|c|}
\hline \multicolumn{1}{|c|}{ Nivel de riesgo } & $\%$ \\
\hline Sin Riesgo & $43 \%$ \\
\hline En riesgo $\left(5 \mu \mathrm{g} / \mathrm{m}^{3}<\mathrm{x}<6 \mu \mathrm{g} / \mathrm{m}^{3}\right)$ & $9 \%$ \\
\hline En alto riesgo $>6 \mu \mathrm{g} / \mathrm{m}^{3}$ & $49 \%$ \\
\hline
\end{tabular}

Tabla III. Cálculo del nivel de riesgo

Fuente: elaboración propia, 2020.

Como se puede evidenciar en la gráfica anterior, durante los primeros días de medición se observa un bajo nivel de exposición a material particulado, debido a que dichas muestras se llevaron a cabo durante la temporada de lluvias, hecho que claramente influyó sobre las concentraciones en comparación con las mediciones finales realizadas en temporada seca. Esto, sumado a las diferentes variables meteorológicas, como la dirección del viento, que es un factor importante dado que la ruta es perpendicular al corredor vial de la calle 26, por donde provienen los vientos de los cerros orientales y en conjunto con la cantidad de edificios con altura considerable (características propias de la ruta), no permiten la dispersión de partículas contaminantes por medio de la circulación y el flujo del aire debido a que actúan como una barrera para detener el material particulado, lo que genera que aumenten las concentraciones al punto de sobrepasar los límites permisibles. 
Con el fin de poder tener una mayor comprensión acerca del por qué se generaban esos niveles tan altos de material particulado, se decidió realizar un análisis por medio del programa estadístico $\mathrm{R}$ para comprender el comportamiento de las variables a través de los modelos lineal y exponencial multivariado.

Para el análisis de los resultados se tuvo en cuenta las siguientes variables:

- Concentración de material particulado ug $/ \mathrm{m}^{3}$

- Humedad del día (promedio porcentual \%)

- Temperatura del día $\left({ }^{\circ} \mathrm{C}\right)$

- Velocidad del viento $(\mathrm{km} / \mathrm{h})$

\section{B. Modelo lineal multivariado}

Con el cual se pretende demostrar la relación de las variables con la concentración de material particulado.

Modelo teórico

$$
y=B 0+B 1 \times 1+B 2 \times 2+B 3 \times 3+e
$$

Donde:

- $\mathrm{x} 1$ : temperatura

- x2: humedad

- x3: velocidad del viento

- y: concentración

En el caso de este modelo ajustado a dichos datos, la temperatura no fue significativa, es decir, no se puede evidenciar una relación entre esta y la variable respuesta que sea importante estadísticamente.

La variable humedad del día fue significativa, lo que indica que esta aporta al modelo y establece una relación de carácter lineal con la variable respuesta, que es la concentración, su valor de estimación es de -15.24 , coeficiente que indica que al aumentar una unidad en la variable humedad cuando la temperatura permanece fija, el cambio en la pendiente de asociación con la concentración es negativo, es decir, se presenta una relación inversa, por lo cual se puede decir que a mayor humedad, menor es la concentración de partículas, y a menor humedad, mayor concentración de partículas.

\section{Modelo exponencial multivariado}

Con el cual se pretende demostrar la relación de las variables con la concentración de material particulado.

$$
\begin{gathered}
y=\exp (B 0+B 1 \times 1+B 2 \times 2+B 3 \times 3+e) \\
\ln (y)=B 0+B 1 \times 1+B 2 \times 2+B 3 \times 3+e
\end{gathered}
$$

Donde:

- x1: Temperatura

- x2: Humedad

- x3: Velocidad del viento

- y: Concentración

En este caso, en el modelo exponencial multivariado ajustado a estos datos, la velocidad del viento no fue significativa, esto significa que no hay suficiente evidencia estadística entre esta variable y la concentración. Las variables humedad del día y temperatura del día fueron significativas, esto muestra que sí aportan al modelo y existe una relación con la variable respuesta, que es la concentración.

El coeficiente estimado para la humedad del día es -2.328487 , este valor indica que a medida que la humedad del día aumenta en una unidad y la temperatura del día y la velocidad del viento permanecen constantes, la concentración de partículas decrece un $9.7443 \%(\exp (-2.328487)=$ 0.097443 ). Por otra parte, el coeficiente estimado para la temperatura del día es 0.169959 , este valor indica que a medida que la temperatura del día aumenta en una unidad y la humedad del día y la velocidad del viento permanecen constantes, la concentración de partículas aumenta un $18.5256 \%$ $(\exp (0.169959)=1.185256)$. Al final de la salida del programa se muestra que el valor R cuadrado de adecuación en el modelo exponencial multivariado es de 0.1998 , esto indica que este modelo explica aproximadamente un $20 \%$ de variabilidad en los datos, es decir no es tan exacto, lo cual sería una posible explicación al por qué es que existen algunos valores atípicos en los datos y que están llegando a afectar la estimación.

Debido a que al porcentaje de $\mathrm{R}$ cuadrado es muy bajo en ambos modelos, se decidió realizar un modelo de dinámica de sistemas, se agregó a este nuevo análisis otras variables que se podrían 
considerar significativas ya que son propias de la ruta y por tal razón podrían llegar a afectar las mediciones de material particulado. Estas son:

- Numero de edificios con una altura significativa (mayor a 5 metros, ya que estos pueden provocar retención de partículas y no permiten que estas fluyan con el viento).

- Número de fuentes de emisión presentes sobre la ruta.

- Porcentaje de vía no asfaltado o en mal estado.

Model: C:IUsers\linav\OneDrive\EscritoriolFIGURA 4 FORRESTER.mdl View: View 1

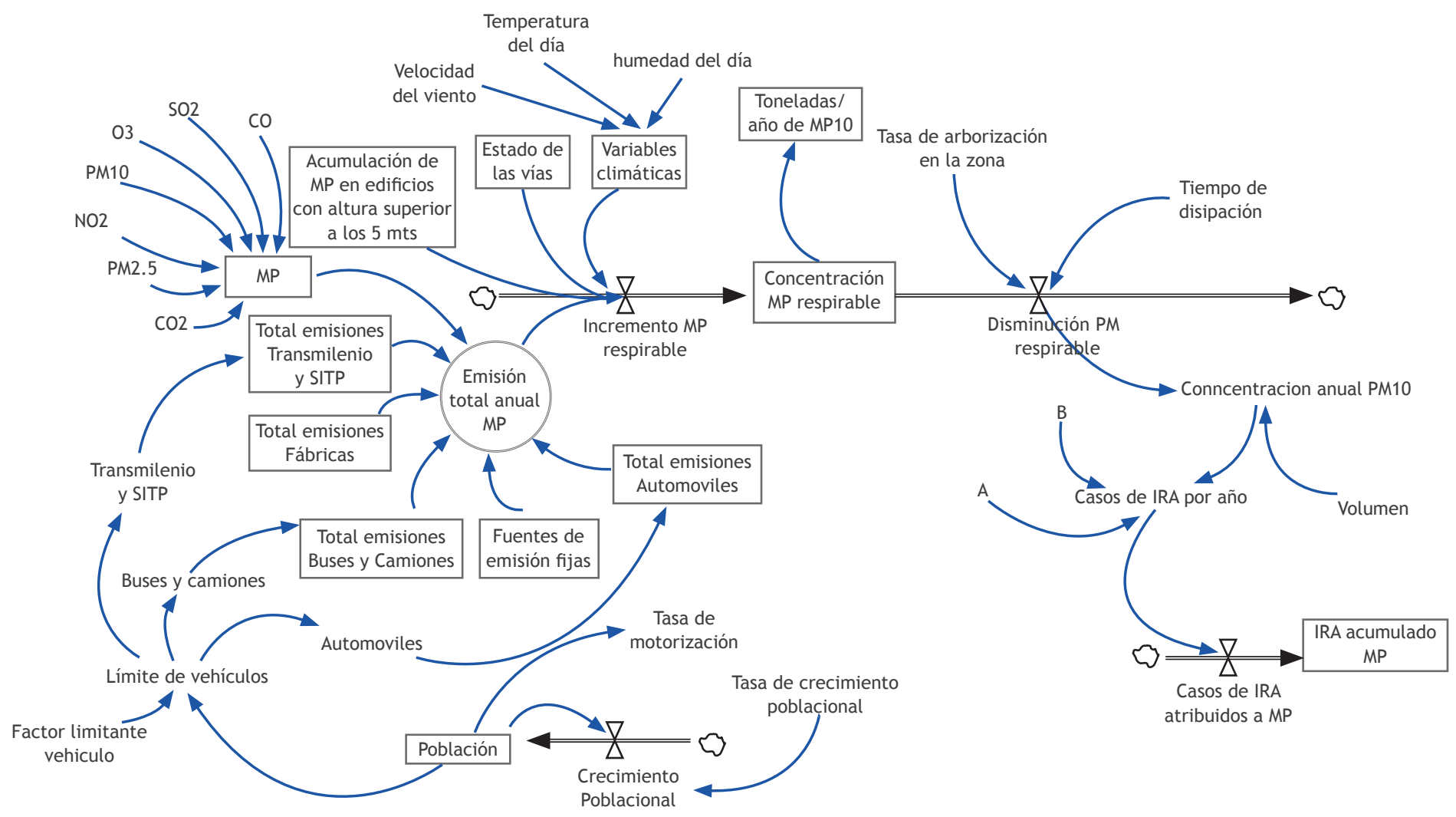

Thu Sep 10, 2020 1:12PM

Figura 4. Diagrama de Forrester - Componente material particulado.

Fuente: elaboración propia, 2020. 


\section{D.Modelo de dinámica de sistemas}

Comúnmente utilizado para analizar el comportamiento de los datos en entornos complejos, como las variables anteriormente descritas y su influencia en las concentraciones de material particulado.

Como se puede evidenciar, se identificaron dos niveles de variables que afectan el nivel de concentración de material particulado en la ciudad, las cuales están conformadas por: el parque automotor (vehículos, buses, Transmilenio, etc.), y las emisiones de las fábricas y demás fuentes, tanto fijas como móviles, que constituyen el dato de emisiones de PM10 (variables auxiliares), además de otras características propias de la ruta que influyen en el incremento del material particulado, como la altura de los edificios, la cual no permite la dispersión de las partículas que se generan en la vía por vehículos que se movilizan en la ruta, la disposición estructural de la vía y las condiciones meteorológicas que pueden influir en el incremento de material particulado. Estas variables de la ruta se describen a continuación:

\begin{tabular}{|l|l|}
\multicolumn{1}{|c|}{ Variable } & \multicolumn{1}{c|}{ Cantidad } \\
\hline Numero de edificios altura $>5 \mathrm{~m}$ & 126 edificios \\
\hline \% ruta no asfaltada & $15 \%$ \\
\hline Fuentes fijas de emisión & 51 fuentes fijas \\
\hline Semáforos & 6 semáforos \\
\hline
\end{tabular}

Tabla IV. Cuantificación de variables propias de la ruta

Fuente: elaboración propia, 2020.

De acuerdo con la información de la tabla anterior se procede a realizar una matriz de correlación de variables que mide las relaciones entre pares de variables y elimina el efecto de las restantes [17].

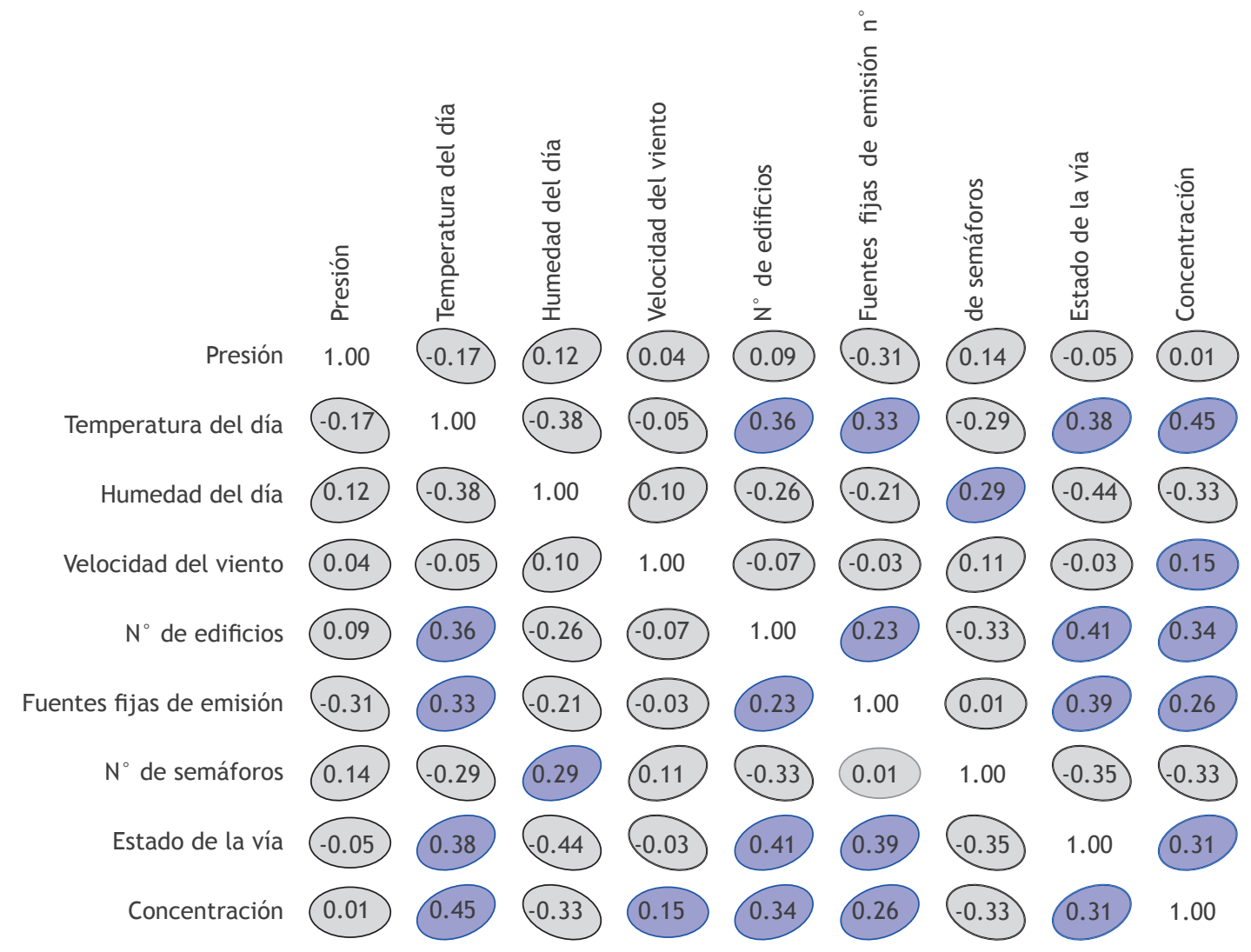

Figura 5. Matriz de correlación de variables. Fuente: elaboración propia, 2020. 
Realizado el análisis con un nivel de confianza de $95 \%$ se logró un mejor entendimiento del funcionamiento del sistema planteado, se identificó que la variable con mayor nivel de relación respecto a la afectación en los niveles de concentración de la muestra de material particulado fue la temperatura, con un porcentaje de correlación del $45 \%$. Seguida de la variable auxiliar denominada número de edificios con altura significativa, la cual obtuvo un $34 \%$ de nivel de correlación, y en tercer lugar está la variable de estado de la vía con un $31 \%$.

Sobre la discusión de los resultados obtenidos se tiene lo siguiente:

1. Se encuentran muy pocos estudios en Colombia sobre esta temática específica, ya que se estudian más las concentraciones de material particulado ambiental y no la fracción respirable.

2. Aunque el modelo de dinámica de sistemas junto con el diagrama de forrester son una buena representación de las múltiples causas que llevan al incremento del material particulado respirable, es necesario que estos se alimenten de nueva información de otras variables que afecten las concentración, tales como las características propias del entorno y la infraestructura vial.

3. Las rutas de movilidad impactan el nivel de riesgo. Esto evidencia que temas como el flujo vehicular en la vía, son vitales en el estudio y más en la ruta, donde se realizó el muestreo, dado que no se cuenta con ciclorruta y el biciusuario podría estar expuesto a concentraciones más altas de material particulado. Esto concuerda con los antecedentes mencionados donde algunos autores señalan que uno de los factores más influyentes en este tipo de investigaciones sería el flujo vehicular y la tipología del vehículo circundante.

4. A futuro es necesario estudiar otros modelos estadísticos aparte de los lineales, ya que los datos meteorológicos analizados en los modelos lineal y exponencial multivariado no son suficientes para determinar el nivel de riesgo por exposición a material particulado, dado que existen diferentes variables propias de la ruta que pueden ser significantes y elevar el nivel de riesgo por exposición a este contaminante.

\section{CONCLUSIONES}

- El muestreo realizado permite concluir que los biciusuarios que transitan por las vías de alto tráfico vehicular están expuestos a material particulado inhalable PM10, el cual genera posibles efectos nocivos en su sistema respiratorio.

- La ruta de estudio no cuenta con ciclorruta y el carril para los ciclistas es compartido con los vehículos circundantes, por esta razón se ven reflejados valores elevados en las concentraciones de PM10 que demuestran que el $49 \%$ de los biciusuarios se encuentran en un nivel de riesgo alto por exposición a este contaminante.

- La determinación de las concentraciones de material particulado respirable por los usuarios es compleja ya que tanto la medición como la toma de muestras se realizan en campos donde existen aspectos no controlados, a diferencia de los que se hacen en espacios laborales con condiciones normales.

- Teniendo en cuenta los resultados obtenidos en los modelos estadísticos se pudo determinar que la variables temperatura y humedad inciden notoriamente en el alto o bajo nivel de concentración de material particulado, de acuerdo con la temporada seca o de lluvias respectivamente.

- A lo largo del estudio se evidenció que la influencia de los vientos, la presencia de edificios de más de 5 metros de altura y otras características propias de la vía, aumentan los niveles de concentración de PM10 debido a la retención de partículas.

- Por todo lo anterior, se hace necesario realizar estudios de higiene industrial aplicados al tema de movilidad en bicicleta, con mayores mediciones, no solo en diferentes rutas de Bogotá, sino también en varias ciudades del país, que permitan conocer el nivel de exposición de los biciusuarios a estos contaminantes y así definir cuáles deberían ser las recomendaciones adecuadas para ayudar a la planeación del viaje en bicicleta, ya que es un tema que está en auge y anualmente se incrementa el número de usuarios de este medio de transporte.

\section{RECONOCIMIENTOS}

Gran agradecimiento a la ingeniera Sonia Meneses y a la Universidad Libre, por su colaboración y contribución para el desarrollo y finalización de este proyecto; al igual que a nuestras familias por su apoyo incondicional para la culminación de este proyecto. 


\section{REFERENCIAS}

[1] R. Ríos, A. Taddia, C. Pardo y N. Lleras, Ciclo-inclusión en América Latina y el Caribe: Guía para impulsar el uso de la bicicleta, Banco Interamericano de Desarrollo, 2015. [En línea]. Disponible en: https://publications.iadb.org/es/ciclo-inclusion-en-america-latina-y-el-caribe-guia-para-impulsar-el-uso-de-la-bicicleta

[2] D. Duarte, K. López y S. Meneses, "Caracterización de riesgos en la accidentalidad de biciusuarios. Bogotá - Engativá”, Revista de Ingeniería Industrial. Actualidad y Nuevas Tendencias, vol. 6, n. ${ }^{\circ}$ 21, pp. 93-108, 2018. [En línea]. Disponible en: http://servicio. bc.uc.edu.ve/ingenieria/revista/Inge-Industrial/vol6-n21/art06.pdf

[3] F. Matt, T. Cole-Hunter, D. Donaire-González, N. Kubesch, D. Martínez, G. Carrasco-Turigas and $M$. Nieuwenhuijsen. "Acute respiratory response to traffic-related air pollution during physical activity performance", Environ Int., vol. 97, pp. 45-55, 2016. [On line]. Available: https://doi.org/10.1016/j.envint.2016.10.011

[4] A. de Nazelle, S. Fruin, D. Westerdahl, D. Martinez, A. Ripoll, N. Kubesch and M. Nieuwenhuijsen, "A travel mode comparison of commuters' exposures to air pollutants in Barcelona", Atmos Environ, vol. 59, pp. 151159, 2012. [On line]. Available: https://doi. org/10.1016/j.atmosenv.2012.05.013

[5] Organización Mundial de la Salud, (2018). "Calidad del aire y salud". [En línea]. Disponible en: https://www.who.int/es/newsroom/fact-sheets/detail/ambient-(outdoor)-air-quality-and-health

[6] A. Thai, I. McKendry and M. Brauer, "Particulate matter exposure along designated bicycle routes in Vancouver, British Columbia", Sci Total Environ, vol. 405, $\mathrm{n}^{\circ}$. 1-3, pp. 2635, 2008. [On line]. Available: https://doi. org/10.1016/j.scitotenv.2008.06.035

[7] M. Montoya-Rendón, P. Zapata-Saldarriaga y M. Correa-Ochoa, "Contaminación ambiental por PM10 dentro y fuera del domicilio y capacidad respiratoria en Puerto Nare, Colombia”, Revista de Salud Pública, vol. 15, $\mathrm{n}^{\circ}$. 1, pp. 103-115, 2013. [En línea]. Disponible en: http://dx.doi.org/10.15446/rsap

[8] Z. Zlatev, K. Georgiev and I. Dimov, "Influence of climatic changes on pollution levels in the Balkan Peninsula". Comput Math with Appl, vol. 65, $\mathrm{n}^{\circ}$. 3, pp. 544-562, 2013. [On line]. Available: https://doi.org/10.1016/j. camwa.2012.07.006

[9] S. Kingham, I. Longley, J. Salmond, W. Pattinson and K. Shrestha, "Variations in exposure to traffic pollution while travelling by different modes in a low density, less congested city", Environ Pollut, vol. 181, pp. 211-218, 2013. [On line]. Available en: https: //doi.org/10.1016/j.envpol.2013.06.030

[10] JF. Franco, "Urban Air Pollution in Bogota, Colombia: An Environmental Justice Perspective", en 105th Air and Waste Management Association Annual Conference and Exhibition, San Antonio, Texas, USA, june 2012. [Online]. Available: https://www.researchgate.net/publication/311327866_Urban_Air_Pollution_in_Bogota_Colombia_an_ Environmental_Justice_Perspective

[11] OA. Fajardo, NY. Rojas, "Particulate matter exposure of bicycle pathusers in a high-altitude city", Atmos Environ, vol. 46, pp. 675679, 2012. [On line]. Available: https://doi. org/10.1016/j.atmosenv.2011.09.047

[12] NIOSH, "Particulates not Otherwise Regulated, Respirable 0600" en Manual of Analytical Methods (NMAM), Fourth Edition.

[13] G. Sánchez, Datos del proyecto. Universidad Libre, Bogotá, 2019.

[14] Icontec, Gestión del riesgo. Bogotá: Icontec, 2016.

[15] Icontec, NTC-IEC/ISO 31010 Técnicas de Valoración de Riesgos, Bogotá: Icontec, 2016.

[16] H. Gutiérrez. (2009). "Cartas de control para variables - Control estadístico de calidad y seis sigma". [Internet]. Disponible en: https: / / issuu.com/mkarina1/docs/3.3_cartas_de_control_para_variable.

[17] J. Marín, Estadística descriptiva y multivariante. [En línea]. Disponible en : http:// halweb.uc3m.es/esp/Personal/personas/ jmmarin/esp/AMult/tema2am.pdf 